\title{
Survival of Vibrio cholerae Inside Acanthamoeba and Detection of Both Microorganisms From Natural Water Samples May Point out the Amoeba as a Protozoal Host for $V$. cholerae
}

\author{
Hadi Abd ${ }^{1 *}$, Salah Shanan ${ }^{1,2}$, Amir Saeed $^{1}$ and Gunnar Sandström ${ }^{1}$
}

${ }^{1}$ Karolinska Institute, Department of Laboratory Medicine, Division of Clinical Microbiology, Karolinska University Hospital, Huddinge, SE-141 86 Stockholm, Sweden ${ }^{2}$ University of Medical Sciences and Technology, Faculty of Medical Laboratory Sciences, Khartoum, Sudan

\begin{abstract}
Summary
The ability of free living and waterborne amoebae to feed on bacteria in the surroundings, as well as to host several human bacteria suggests that both amoebae and bacteria are involved in complex interactions. The extracellular bacterium, Vibrio cholerae requires $10^{8}$ to $10^{9}$ cells to cause cholera, and accordingly it needs an environmental host to grow to such high numbers to be able to cause the infection in humans. The current review discusses the properties of $V$. cholerae to be able to grow inside the environmental protozoa Acanthamoeba species, findings of our field study applied molecular detection of both microorganisms in the same natural water samples from cholera endemic area, and role of Acanthamoeba as a protozoal host to $V$. cholerae in nature beside human.
\end{abstract}

Keywords: Cholera; Natural water; Acanthamoeba; Second host; V. cholerae; Intracellular survival

\section{Introduction}

Vibrio cholerae is a gram-negative bacterium and it known to cause cholera. Cholera is an acute diarrhoeal disease that can kill within hours if left untreated. The actual global disease burden is estimated to be 3-5 million cases and 100 000-130 000 deaths per year [1]. V. cholerae and free-living amoebae (FLA) are present in aquatic environments, including drinking water $[2,3,4]$.

Acanthamoeba is a genus of FLA, which are environmental eukaryotic cells distributed worldwide in nature [5,6] and they are found to support bacterial growth and survival [5]. Recently, studies have shown that Pseudomonas aeruginosa kills A. castellanii [7] and Aeromonas hydrophila inhibits growth of A. castellanii [8]. Interestingly, V. cholerae $\mathrm{O} 1, \mathrm{O} 139$ and $V$. mimicus have grown and survived inside Acanthamoeba species [9-14].

Since ability of different bacteria to interact differently with FLA needs to disclose mechanisms of the interactions, which are important to the medical- and environmental microbiology, the current review discusses findings about growth and survival of $V$. cholerae inside Acanthamoeba castellanii and A. polyphaga, detection of both microorganisms in the same natural water samples from cholera endemic area, and role of Acanthamoeba as an environmental host to V. cholerae.

\section{Biology and ecology of $V$. cholerae}

Vibrio is a genus of Gram-negative bacteria that comprise nearly 70 species [15] such as $V$. cholerae, $V$. parahaemolyticus and $V$. mimicus. These bacteria found in water and they can be carried by sea living animals, such as shellfishes. The prevalence rate of infections caused by Vibrio species appears to be increasing globally. The combination of increased water temperature and salinity may contribute to increased association rates of the bacteria with sea living animals or protozoa. In the event of a natural disaster, the disturbance to the environment may increase the risk of infectious diseases such as Vibrio infections. Vibrio species can produce multiple extracellular cytotoxins and enzymes that are associated with extensive tissue damage and that may play a major role in the development of sepsis [16].
$V$. cholerae includes more than 200 serogroups based on the $\mathrm{O}$ antigenic structures [17]. Only serogroup O1 and O139 possess cholera toxin gene and cause epidemic and pandemic cholera that affects many millions annually creating a worldwide health problem [18-21]. Non-O1/O139 V. cholerae are serologically diverse strains, which are abundant in estuarine environments. They are sporadically involved in cholera-like diarrheal disease [22-26], but rarely in outbreaks. $V$. cholerae $\mathrm{O} 141$ and $V$. cholerae $\mathrm{O} 75$ possess cholera toxin gene but not produce cholera toxin $[27,28]$.

Vibrio mimicus shares similar properties with $V$. cholerae such as existence of virulence associated genes, namely cholera toxin as well as toxin co-regulated pilus genes [29] and both species possess LuxO protein that regulates protease activity [30].

$V$. cholerae species are straight or curved rods widely distributed in aquatic environments [31]. Evidence suggests that $V$. cholerae is a component of the autochthonous flora of brackish water, estuaries, and salt marshes of coastal areas of the temperate zone, posing an ongoing hazard to public health $[32,33]$. Various $V$. cholerae O1 strains have become endemic in many regions in the world, including Australia and the Gulf Coast region of the US [20,34].

Cholera outbreaks are thought to have resulted from consumption of raw, undercooked, contaminated, or re-contaminated seafood since $V$. cholerae is transmitted primarily by the faecal-oral route, indirectly through contaminated water supplies $[23,33,35,36,37,38]$.

${ }^{*}$ Corresponding author: Dr. Hadi Abd, Karolinska Institute, Department of Laboratory Medicine, Division of Clinical Microbiology, Karolinska University Hospital, Huddinge, SE-141 86 Stockholm, Sweden, Tel:076 21496 30; E-mail: hadi.abd@ki.se

Received November 08, 2011; Accepted December 09, 2011; Published December 15, 2011

Citation: Abd H, Shanan S, Saeed A, Sandström G (2011) Survival of Vibrio cholerae Inside Acanthamoeba and Detection of Both Microorganisms From Natural Water Samples May Point out the Amoeba as a Protozoal Host for V. cholerae. J Bacteriol Parasitol S1-002. doi:10.4172/2155-9597.S1-003

Copyright: (c) $2011 \mathrm{Abd} \mathrm{H}$, et al. This is an open-access article distributed under the terms of the Creative Commons Attribution License, which permits unrestricted use, distribution, and reproduction in any medium, provided the original author and source are credited. 
Citation: Abd H, Shanan S, Saeed A, Sandström G (2011) Survival of Vibrio cholerae Inside Acanthamoeba and Detection of Both Microorganisms From Natural Water Samples May Point out the Amoeba as a Protozoal Host for V. cholerae. J Bacteriol Parasitol S1-003. doi:10.4172/21559597.S1-003

Food supplies may be contaminated by the use of human faeces as fertilizer or by freshening vegetables for market with contaminated water $[17,35,39,40,36]$. Any infected water and any foods washed in that water, as well as shellfish living in the affected waterway, can cause an infection. The causative agent of cholera is rarely spread directly from person to person.

$V$. cholerae harbors naturally in the zooplankton of fresh, brackish, and salt water, attached primarily to their chitinous exoskeleton [41]. The bacterium has been isolated routinely from many aquatic environments throughout the world, often in association with plankton, plants, invertebrates, and fish, and there are some reports of its presence in water birds, seals, and diseased farm animals [42].

Despite amoebae are significant predators of bacteria in soil and aquatic environments [43], many biological factors affect the survival of $V$. cholerae in aquatic environments such as loss to predators [44] and regulating the level of viable cells of $V$. cholerae [45] to be able to infect humans. The dose of $V$. cholerae needed to cause cholera is approximately $10^{8}$ cells [21]. Therefore, the bacteria need a biological reservoir as training ground in order to grow and survive in concentrations high enough to infect humans. It has been stated that humans are the only known host and reservoir for $V$. cholerae outside its aquatic environment [46] but according to the need of high numbers of bacteria to cause infection it seems likely that the bacterium has an environmental host beside man and that such a host may support growth and survival of the bacterium in nature. Finding of aquatic reservoirs of $V$. cholerae is an important factor in the epidemiology of cholera.

\section{Amoebae predators or hosts for bacteria but special bacteria kill the amoebae}

FLA are eukaryotic cells found in nature [5] and including several genera such as Acanthamoeba, Balamuthia, Naegleria[6] Sappinia [47] and Dictyostelium [48]. Association of Dictyostelium was studied with a number of pathogenic bacteria for more than hundred years ago since the role of the accompanying bacteria rightfully occupied the center of attention [49]. Interestingly, Raper and Smith (1939) found an excellent growth of $D$. discoideum with Escherichia coli and a suppressed growth with $P$. aeruginosa compared to good growth occurred in association with Shigella dysenteriae, Salmonella enteritis and S. paratyphi [49]. Moreover, Thom et al., 1992 showed that $V$. cholerae could grow and survive during $24 \mathrm{~h}$ in microcosms pre-inoculated with trophozoites of freshwater amoebae [50]

In this context, it has been shown that Acanthamoebae benefit from E. coli and Klebsiella aerogenes as food [51]. In contrast, the role of Acanthamoebae as hosts for bacteria, has been proposed for many pathogenic bacteria, for example Campylobacter jejuni [52], Coxiella burnetii [53], E. coli O157 [54], E. coli K1 [55], Francisella tularensis [56], Helicobacter pylori [57], Legionella pneumophila [58], Listeria monocytogenes [59], Mycobacterium avium [26], S. dysenteriae [25], S. sonnei $[60,61,25]$ and S. typhimurium [62]. The Acanthamoebae support bacterial growth and survival and save the bacteria from chlorination [63] increasing the risk of human illness caused by bacteria or Acanthamoebae. However, Fritsche et al. [64] has found that $25 \%$ of environmental and clinical Acanthamoeba species isolates contain obligate bacterial endosymbionts.

Why some bacteria are utilised as food by the amoebae and other bacteria utilised the amoebae as hosts, and why a number of special bacteria kill the amoeba!
Generally, bacteria can be divided into intracellular and extracellular bacteria according to avoid phagocytosis or not and to multiply inside or outside the host cells [65]. Many bacteria possess type three secretion system (TTSS) other bacteria do not. These differences between bacteria may affect their interaction with the amoebae.

TTSS is found in many gram-negative pathogenic bacteria, such as Yersinia spp., P. aeruginosa, Salmonella spp., Shigella spp., enteropathogenic, enterohemorrhagic and neuropathogenic E. coli strains. TTSS consists of structural-, effectors proteins and chaperones. The structural proteins build the apparatus consisting of base, inner rod and needle. The needle translocates effector proteins into the plasma membranes to inject the effector proteins into the cytoplasm of eukaryotic cells to promote infection in target cells. The chaperones bind the effectors in the bacterial cytoplasm, protect them from aggregation and degradation and direct them towards the needle complex [84]. TTSS plays a key role in the bacterial pathogenicity and infection; therefore, output of extracellular and intracellular bacteria that possess or lack TTSS, on interaction with amoebae was studied by the researchers.

It has been found that the extracellular bacterium $P$. aeruginosa possessing TTSS injects four different effector proteins (exoenzymes) described as ExoS, ExoT, ExoU, and ExoY. These effector proteins are able to inhibit DNA synthesis as well as phagocytosis in the host cell and to induce necrosis and cell lysis associated with cell injury damage and dissemination of $P$. aeruginosa within infected hosts [7].

Abd et al., studied the interaction between A. castellanii and $P$. aeruginosa and the result has shown that neither the presence nor absence of $A$. castellanii has any effect on growth of $P$. aeruginosa, since large numbers of bacteria remain outside the amoebae during the interaction, revealing $P$. aeruginosa to be strictly extracellular bacteria growing better outside than inside eukaryotic cells. However, the encystation of $A$. castellanii can be stimulated by either wild type $P$. aeruginosa possessing different TTSS effector proteins or TTSS-deficient strains. Interestingly, wild type $P$. aeruginosa strains kill $A$. castellanii, while TTSS deficient strains inhibit growth of the amoebae. Thus, stimulation of encystation and inhibition of amoebic growth might be caused by the extracellular virulence factors such as extracellular factors produced by the Rhl quorum sensing system. $P$. aeruginosa wild type or deficient in TTSS effector proteins can cause necrosis and apoptosis in amoebae, but TTSS effector proteins of the wild type are also capable of lysing more amoeba cells, resulting in decreased numbers of necrotic, apoptotic and normal amoeba cells. The difference between the effect of strains possessing TTSS effector proteins and strains deficient in these proteins on $A$. castellanii is clearly the difference between killing and inhibition [7]. In this context, It has been reviewed that $P$. aeruginosa utilises TTSS effector proteins to kill macrophages, epithelial cells and the amoeba D. discoideum, as is also the case with A. castellanii [7]. Thus, TTSS is a potent virulence factor for the extracellular bacterium $P$. aeruginosa and $A$. castellanii represents a novel model to study wild type bacteria and parental mutants in TTSS.

In comparison, it was found that the extracellular and nonpathogenic E. coli K12 lacking TTSS had no effect on A. castellanii viability and the bacterium failed to grow inside the amoeba cells [55]. However, co-cultivation of the facultative intracellular neuropathogenic E. coli $\mathrm{K} 1$ and $E$. coli $\mathrm{K} 12$ associated with $A$. castellanii showed that E. coli $\mathrm{K} 1$ lysed the amoebae and grew exponentially, whereas $E$. coli K-12 exhibited minimal growth [85]. Surprisingly, Siddiqui et al., has demonstrated recently that the neuropathogenic E. coli K1 strains 
Citation: Abd H, Shanan S, Saeed A, Sandström G (2011) Survival of Vibrio cholerae Inside Acanthamoeba and Detection of Both Microorganisms From Natural Water Samples May Point out the Amoeba as a Protozoal Host for V. cholerae. J Bacteriol Parasitol S1-003. doi:10.4172/21559597.S1-003

possesses TTSS and the deletion mutant of TTSS in E. coli exhibits defects in the invasion and intracellular survival in A. castellanii [85].

F. tularensis is a gram-negative bacterium causing tularemia in humans and animals. Tularemia is a zoonotic disease, which results in fever, rash and swollen lymph nodes. The transmission of the bacterium occurs by several modes such as bites by infected ticks, flies or mosquitoes, or intake of contaminated water, food or soil as well as inhalation of aerosol containing the bacteria and other direct contact means such as handling of tissues or fluids from infected animals. Ecology of the bacterium has shown that it has been isolated from more than 250 animal species such as hares, rabbits and rodents and it can be recovered from contaminated water and soil but its principal natural reservoir is unknown. It is suggested that the bacterium can persist in water courses, possibly in association with amoebae [56].

F. tularensis is facultative intracellular bacterium lacking TTSS [86]. It has potential ability to grow and survive intracellularly in $A$. castellanii. F. tularensis cells infect trophozoites of the amoeba, replicate and grow in membrane bounded vacuoles inside the trophozoites. Cell organelles of the amoeba such as mitochondria and endoplasmic reticulum are recruited to the vacuoles containing bacteria. Infected trophozoites are found both as intact viable cells filled with vacuoles containing $F$. tularensis and, in the process of cytolysis, excreting vesicles containing $F$. tularensis. Some infected trophozoites are also seen undergoing encystations and $F$. tularensis cells can be found in precysts and in mature cysts. The infection process of $F$. tularensis in $A$. castellanii seems to display many features in common with Legionella infection in A. castellanii and in macrophages [56].

The ability of F. tularensis to survive in trophozoites of A. castellanii and its cysts may have implications for the mode of transmission of the microorganism. The close connection of tularaemia with water and the isolation of the bacterium from water samples used for domestic purposes, as well as from natural water systems, support the hypothesis that amoebae play a role in the natural transmission of the facultative intracellular/lacking TTSS F. tularensis. The model of non-mammalian cells and waterborne bacteria can be useful in the search for biological methods to inhibit transmission of pathogens from water to humans.

The interaction of TTSS possessing or lacking extracellular bacteria (P. aeruginosa or E. coli K12) and that of TTSS possessing or lacking intracellular bacteria (E. coli $\mathrm{K} 1$ or F. tularensis) is reviewed in this paper. Next, the reviewed outputs of the extracellular and intracellular bacteria that possess or lack TTSS, on interaction with amoebae will be compared to that of $V$. cholerae O1 and O139 lacking TTSS [84]. $V$. cholerae is a free-living cell in aquatic environment [66] and it is held to be an extracellular bacterium $[4,67]$. What will happen if $V$. cholerae is cultivated with the environmental phagocyte Acanthamoeba castellanii or A. polyphaga in a liquid microcosm? Theoretically, it is expected that $V$. cholerae multiply in the culture liquid and not inside Acanthamoeba cells because it is an extracellular bacterium. But how does the experiment answer this question?

\section{Enhanced growth, viability and survival of $V$. cholerae and $V$.} mimics by co-cultivation with Acanthamoeba species

The interaction of $A$. castellanii or A. polyphaga with $V$. cholerae $\mathrm{O} 1, V$. cholerae $\mathrm{O} 139$ and $V$. mimicus was studied by cultivation of each microorganism alone and by cultivation of each bacterial species or serogroup with the amoebae for two weeks. Growth of the microorganisms was estimated by viable counts and the bacterial localisation in amoebae was disclosed by microscopy.
It was found that the number of alone- and co-cultivated amoeba increased tenfold after one week. Irrespective of $V$. cholerae species or serogroup present the growth of the amoebae was not inhibited [9-14]. The growth of co-cultivated V. cholerae O1 classical and El Tor biogroups, V. cholerae $\mathrm{O} 139$ and $V$. mimicus with amoebae was enhanced and they remained viable under the performed experimental time. The presence of the amoebae enhanced survival of co-cultivated Vibrio species during two weeks, while all these species in the absence of amoebae died within few days and did not enter viable and nonculturable state [9-14].

It was shown that growth of wild type $V$. cholerae O139 MO10, capsule mutant strain, and capsule + lipopolysaccharide (LPS) double mutant strain were enhanced in the presence of A. castellanii [10]. Previous studies have shown that both capsule and LPS O side chain of $V$. cholerae MO10 enhance adherence to the human intestinal mucosa $[68,69]$ and the capsule may contribute to partially resist phagocytosis [70].

In comparison to macrophages, Acanthamoebae utilise different mechanisms to capture bacteria by means of specific and non-specific adherence as well as food cup formation. It is well known that $A$. castellanii takes up bacteria by pseudopodia to form food vacuoles in which phagocytosis and digestion occur within phagolysosomes [24] or by food cup formation and ingestion of particulate matter [71] Adherence of bacteria to eukaryotes is the first step in interaction between bacteria and host cell [72]. Bacterial adherence to various surfaces includes several manners, including hydrophobic and ionic bonds and also lectin-like interactions between bacterial ligands and complementary molecules of the substrate or receptors on eukaryotes [73,74]. Lock et al., 1987 found that bacteria possessing mannosesensitive fimbriae were able to adhere to Acanthamoebae and to leucocytes and that addition of mannose inhibited completely bacteria adherence to leucocytes, whereas, it inhibited only partly adherence to Acanthamoebae [73].

It is known that mannose-sensitive haemagglutinin fimbria of $V$. cholerae $\mathrm{O} 139$ contributed to its attachment to plankton in aquatic habitats [75]. In this context, Abd et al., 2009 showed that the addition of mannose neither affected adherence nor uptake and intracellular growth of $V$. cholerae MO10 in A. castellanii [10].This supports the previous finding by Lock et al., 1987 [73] and may indicate that specific adherence mediated by other factors such as outer membrane protein, toxin co-regulated pilus, beside non-specific adherence participate in the interaction between $V$. cholerae and A. castellanii.

A. castellanii and $V$. cholerae lived together in the microcosm. The co-existence of these microorganisms did not inhibit growth of amoebae but enhanced growth, viability and survival of the bacteria instead. How did the enhanced bacterial growth, viability and survival occur?

\section{Intracellular behaviour of $V$. cholerae and $V$. mimicus as a new property}

To answer the previous question, the ability of $V$. cholerae and $\mathrm{V}$. mimics to grow and survive inside A. castellanii or A. polyphaga was investigated by gentamicin treatment to kill extracellular bacteria from co-culture and by sodium deoxycholate solution treatment, which permeabilities amoebae to release intracellular bacteria. The outcome of the treatments was detected by viable counts and visualized by fluorescence microscopy and the localization of intracellular occurring bacteria was visualized by electron microscopy. 
Citation: Abd H, Shanan S, Saeed A, Sandström G (2011) Survival of Vibrio cholerae Inside Acanthamoeba and Detection of Both Microorganisms From Natural Water Samples May Point out the Amoeba as a Protozoal Host for V. cholerae. J Bacteriol Parasitol S1-003. doi:10.4172/21559597.S1-003

Page 4 of 8

Surprisingly intracellular growth of $V$. cholerae and $V$. mimicus occurred and the viable counts of the intracellular growing bacteria showed an increase in the number from non-detectable levels on day 0 to $10^{3}$ and $10^{5} \mathrm{CFU} / \mathrm{ml}$ on day 1 and 14 , respectively [9-14]. The intracellular localisation of $V$. cholerae strains in A. castellanii was confirmed by fluorescence microscopy and electron microscopy. The fluorescence microscopic analysis confirmed viability of intracellular $V$. cholerae $\mathrm{O} 1$ tagged green fluorescent protein (GFP), which emits green fluorescence (Figure 1B), compared to A. castellanii cells, which emits red auto fluorescence (Figure 1A). To know whether the fluorescent bacteria entered viable but non-culturable state or not, the viable count assay confirmed that the bacteria are viable and culturable because they grew on agar plates, and the growing colonies emitted green fluorescence also when exposed to ultraviolet light (Figure 1C). Electron microscopy confirmed the intracellular localisation of $V$. cholerae cells in the trophozoites (Figure $2 \mathrm{~B}$ ) as well as in the cysts of A. castellanii (Figure 2C) compared to the trophozoite that did not contain bacteria (Figure 2A).

Trophozoites of $A$. castellanii undergo encystation as a vital, protective, and reversible process to face adverse conditions such as changes in $\mathrm{pH}$, temperature, and food deprivation [76]. However, our studies showed that encystation of $A$. castellanii in presence or absence of $V$. cholerae did not affected by intracellular internalisation of the bacteria [12] in contrast to interaction of A. castellanii with the extracellular bacterium $P$. aeruginosa, which killed the amoebae [7]. Thus, output of different interactions between $V$. cholerae, P. aeruginosa and $A$. castellanii indicated adaption of $V$. cholerae to the intracellular milieus and symbiotical relation between $V$. cholerae and $A$. castellanii.

Despite $V$. cholerae is considered as an extracellular bacterium [4] our studies show that $V$. cholerae has interacted with the environmental phagocyte $A$. castellanii as a facultative intracellular bacterium, similar to F. tularensis [56].

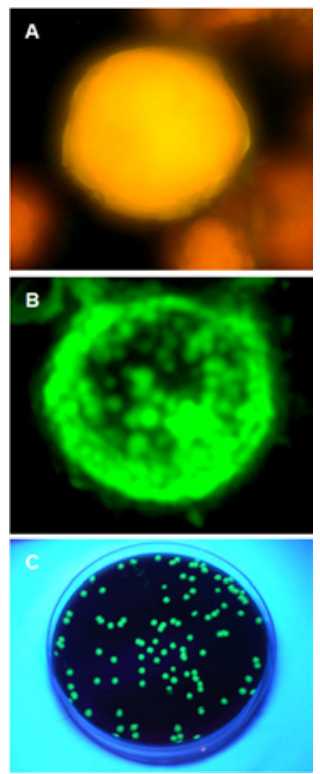

Figure 1: Viability and cultivability of the intracellular $V$. cholerae 01 tagged green fluorescent protein (GFP). A. Alone cultivated Acanthamoeba castellanii cyst emitting autoflourescene. B. Intracellular $V$. cholerae emitting green fluorescence. $C$. The intracellular $V$. cholerae grew on blood agar plate in presence of ampicillin. The colonies emitted green fluorescent when exposed to UV light.

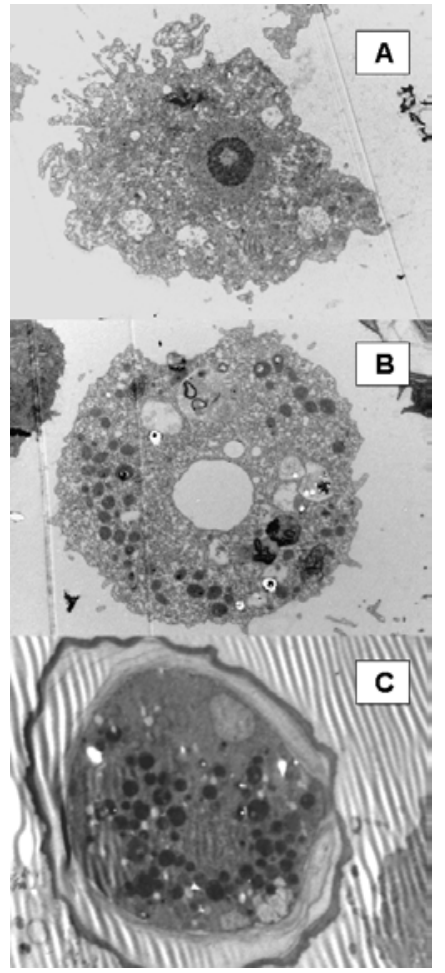

Figure 2: Localisation of $V$. cholerae in $A$. castellanii with a magnification of 3700x.

A. alone cultivated $A$. castellanii trophozoite as negative control, characterized by nucleus, vacuoles and pseudopodia. B. Many empty vacuoles, pseudopodia and more than 50 cells of $V$. cholerae 0139 inside $A$. castellanii trophozoite on day 6 of co-cultivation. $C$. More than 40 cells of $V$. cholerae 0139 inside $A$. castellanii cyst characterized by double wall called ecto-and endocyst, on day 7 of co-cultivation.

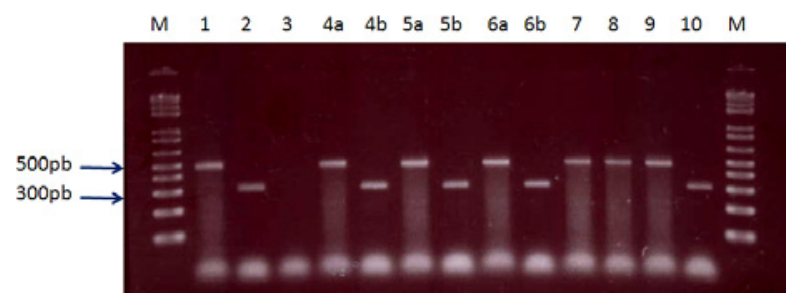

Figure 3: Agarose gel electrophoresis of PCR products of cholera toxin gene (ctxA) and Acanthamoeba 18S rDNA gene. $M$ are molecular mass markers (1500 bp), lane 1 amoebic positive control (approximately 450 bp), 2 bacterial positive control (308bp), 3 negative control, 4 to 6 samples contain both Acanthamoeba (a) and V. cholerae (b), 7 to 9 samples contain amoebae only, 10 sample containing $V$. cholerae only.

It is well known that extracellular bacteria, such as Corynebacterium diphtheriae, Pseudomonas species [46], and Bacillus anthracis [77], are sensitive to phagocytosis; therefore, they may benefit from killing phagocytes before they are ingested [38]. We have found that the extracellular bacterium $P$. aeruginosa induced both apoptosis and necrosis in A. castellanii [7], which is in contrast to the facultative intracellular bacterium, L. pneumophila that does not induce apoptosis in another species of Acanthamoeba, A. polyphaga [78].

The growth pattern of $P$. aeruginosa PA103 in the absence or presence of amoebae [7] indicates that the behaviour of this extracellular bacterium differs from the behaviour of facultative 
Citation: Abd H, Shanan S, Saeed A, Sandström G (2011) Survival of Vibrio cholerae Inside Acanthamoeba and Detection of Both Microorganisms From Natural Water Samples May Point out the Amoeba as a Protozoal Host for V. cholerae. J Bacteriol Parasitol S1-003. doi:10.4172/21559597.S1-003

intracellular bacteria, such as F. tularensis [56] and from V. cholerae that has shown a facultative intracellular behaviour since the growth of $V$. cholerae is enhanced in the presence and inhibited in the absence of amoebae [9-14].

Behaviour of $V$. cholerae differs also from other strict extracellular bacteria, such as $E$. coli and $K$. aerogenes, which provide excellent nourishment for $A$. castellanii and A. polyphaga $[55,51]$.

Growth pattern of $V$. cholerae $\mathrm{O} 1$ strains showed that numbers both of amoebae and intracellular bacteria increased over the experimental time, and the statistical analysis showed that the growth of intracellular bacteria is dependent on the growth of amoebae in a strong positive linear relation; R2 was 0.87 [12]. The behaviour of $V$. cholerae $\mathrm{O} 1$ and $V$. cholerae $\mathrm{O} 139$ in our studies $[10,12-14]$ resembles the behaviour of the facultative intracellular bacterium $F$. tularensis [56] when it interacts with $A$. castellanii but differs from the behaviour of the extracellular bacterium $P$. aeruginosa. Recent study showed that $P$. aeruginosa grew to $10^{9} \mathrm{CFU} / \mathrm{ml}$ on day 1 , in spite of different multiplicities of infection (MOIs) when this bacterium was cultured alone or with amoebae, and that both alone and co-cultivated $P$. aeruginosa survived at the same level during the experimental time, which was 12 days [7]. In comparison, growth and survival of $F$. tularensis [56] as well as $V$. cholerae $[10,12-14]$ were enhanced in the presence and inhibited in the absence of amoebae.

The common property of toxigenic $V$. cholerae serogroups is the presence of toxin-co-regulated pilus (TCP), which is a colonization factor to the intestine of humans [31]. V. cholerae O1 El Tor and O139 possess mannose-sensitive haemagglutinin (MSHA), which is required for colonization to zooplankton [75]. However, it is well documented that $V$. cholerae $\mathrm{O} 139$ possesses a capsule whereas $\mathrm{O} 1$ strains do not $[79,80]$.

Although there are differences between toxigenic $V$. cholerae serogroups and biotypes, our studies $[10,12-14]$ showed that $V$. cholerae $\mathrm{O} 1$ and $\mathrm{O} 139$ grow and survive intracellularly in A. castellanii. These findings confirm a facultative intracellular behavior of $V$. cholerae strains as a new common property, which is previously unknown.

The new behaviour of $V$. cholerae to behaveas intracellular bacteria with $A$. castellanii did enhance growth and viability of the cocultivated bacteria and protected the intracellularly $V$. cholerae from antibiotics [10,12-14]. Moreover, growth and viability of the amoebae did not affected, indicating that free-living amoeba acts as host to $V$. cholerae in nature in addition to man. In this context, do the amoebae hosting intracellular $V$. cholerae play a role as biological factor in the epidemiology of cholera?

\section{Enhanced growth of $V$. cholerae to higher number than needed to cause cholera by re-cultivation of $A$. castellanii hosting intracellular $V$. cholerae}

To examine the role of amoebae harbouring intracellular $V$. cholerae as biological factor in the epidemiology of cholera, A. castellanii was cultivated with $V$. cholerae for 1 week. After gentamicin killing and washing of extracellular $V$. cholerae, the number of viable intracellular bacteria as well as viable amoebae was estimated by viable count of bacteria and viability of amoeba cells by using erythrosine staining.

It was found that $4 \times 10^{5}$ cell $/ \mathrm{ml} \mathrm{A}$. castellanii harbouring $2 \times 10^{5}$ cell $/$ $\mathrm{ml}$ intracellular $V$. cholerae was re-cultivated in $50 \mathrm{ml}$ ATCC medium 712 for 2 weeks. The result showed an enhanced growth of $A$. castellanii to $2.3 \times 10^{6} \mathrm{cell} / \mathrm{ml}$ and $V$. cholerae to $2 \times 10^{11} \mathrm{CFU} / \mathrm{ml}$ and both microorganisms survived for more than 2 weeks. In this experiment the amoeba works as a biological incubator where intracellular growth occurred under 4 days to increase number of the bacteria one million fold and maintains overall viability of $V$. cholerae during 2 weeks [81].

$V$. cholerae shows the facultative intracellular bacterial behaviour, because it is found intracellularly as well as extracellularly in the culture medium, which increases the number of the bacteria over the infection dose stipulated for humans. Thus, the ability of $V$. cholerae to grow and survive intracellularly in A. castellanii presented in our studies disclosed one of the biological factors that could enhance the survival of $V$. cholerae in aquatic environments. The free-living amoeba is a possible biological factor, which enhances growth of the bacteria in water and may thereby increase the probability to infect human with cholera $[12,81]$.

\section{Detection of both $V$. cholerae and Acanthamoeba in same natural water samples from cholera endemic area}

$V$. cholerae and Acanthamoeba species are present in aquatic environments, including drinking water [2-4]. A number of studies report that FLA support survival of pathogenic bacteria [5] and more studies are still needed on distribution of $V$. cholerae and FLA in nature [82]

A molecular method was developed to detect $V$. cholerae and A. castellanii if found together in the same environmental water samples, by using primers targeting cholera toxin gene (ctxA) and Acanthamoeba $18 \mathrm{~S}$ rDNA gene [14]. Recently this method was utilised to detect presence of $V$. cholerae and Acanthamoeba species in same natural water samples collected from endemic areas in Sudan. It was found that eight samples contained both $V$. cholerae and Acanthamoeba species. Furthermore, it was found that one sample contained just $V$. cholerae compared to 13 samples which contained Acanthamoeba only. Surprisingly, the detected number of together- and alone-identified amoebae and bacteria was significantly differed (pvalue of $\chi 2$ was < $0.05)$. $V$. cholerae needs to be found with other microorganisms such as Acanthamoeba a finding disclosed by this study since $89 \%$ of detected $V$. cholerae was found with Acanthamoebae compared to $11 \% \mathrm{~V}$. cholerae, which was found alone [83]. For the first time this study showed that both $V$. cholerae and Acanthamoeba species can be detected in the same natural water samples collected from different cholera endemic areas in Sudan. Taken together role of Acanthamoeba species in survival of $V$. cholerae [10,12-14] may strongly disclose Acanthamoeba as a biological factor enhancing survival of $V$. cholerae in nature.

\section{Conclusions}

Output of the interaction between bacteria and amoeba is depending on whether the interacted bacterium is an extracellular or intracellular and if it possesses TTSS or not since TTSS effector proteins are observed to affect strongly output of the interaction.

Extracellular bacteria cannot multiply inside amoeba cell. TTSS possessing extracellular bacteria such as $P$. aeruginosa kill the amoeba compared to E. coli that does not possess TTSS is ingested as food by the amoeba.

Intracellular bacteria multiply inside amoeba cell. The intracellular bacterium that does not possess TTSS $F$. tularensis multiplies symbiotically inside the amoebae, while TTSS possessing intracellular bacterium E. coli $\mathrm{K} 1$ lyses the amoebae according to activation of TTSS.

The ability of $V$. cholerae to grow and survive inside $A$. castellanii 
Citation: Abd H, Shanan S, Saeed A, Sandström G (2011) Survival of Vibrio cholerae Inside Acanthamoeba and Detection of Both Microorganisms From Natural Water Samples May Point out the Amoeba as a Protozoal Host for V. cholerae. J Bacteriol Parasitol S1-003. doi:10.4172/21559597.S1-003

Page 6 of 8

confirms that $V$. cholerae strains have a facultative intracellular behaviour as a new property, previously unknown.

The intracellular growth as well as survival of the bacteria does not inhibit growth and viability of amoeba cells indicating a symbiotic relationship between $V$. cholerae and $A$. castellanii.

A. castellanii enhances growth and survival of $V$. cholerae and protects the intracellular bacteria from antibiotic effects, in addition to detection both microorganisms from same natural water samples, supporting the role of amoebae as environmental hosts for $V$. cholerae.

A. castellanii is a suitable model in research to differentiate between extracellular and intracellular bacteria, since the intracellular bacteria are able to multiply inside the amoeba while the extracellular bacteria do not.

\section{References}

1. World health Organization (2011) Cholera. Fact sheet No. 107

2. Brown MR, Barker J (1999) Unexplored reservoirs of pathogenic bacteria: protozoa and biofilms. Trends Microbiol 7: 46-50

3. Backer H (2002) Water disinfection for international and wilderness travellers Clin Infect Dis 34: 355-364.

4. Greub G, Raoult D (2004) Microorganisms resistant to free-living amoebae Clin Microbiol Rev 17: 413-433.

5. Brown TJ, Cursons RT, Keys EA (1982) Amoebae from antarctic soil and water. Appl Environ Microbiol 44: 491-493.

6. Martinez AJ, Visvesvara GS (1997) Free-living, amphizoic and opportunistic amebas. Brain Pathol 7: 583-598.

7. Abd H, Wretlind B, Saeed A, Idsund E, Hultenby K, et al. (2008) Pseudomonas aeruginosa utilises its type III secretion system to kill the free-living amoeba Acanthamoeba castellanii. J Eukaryot Microbiol 55: 235-243.

8. Rahman M, Abd H, Sandström G, Romling U, Möllby R (2008) AeromonasAcanthamoeba interaction and early shift to a viable but non-culturable state of Aeromonas by Acanthamoeba. J Appl Microbiol 104: 1449-1457.

9. Abd H, Valeru SP, Sami SM, Saeed A, Raychaudhuri S, et al. (2010) Interaction between Vibrio mimicus and Acanthamoeba castellanii. Environ Microbio Reports 2: 166-171.

10. Abd H, Saeed A, Weintraub A, Sandström G (2009) Vibrio cholerae 0139 requires neither capsule nor lipopolysaccharide $\mathrm{O}$ side chain to grow inside Acanthamoeba castellanii. J Med Microbiol 58: 125-131.

11. Sandström G, Saeed A, Abd H (2010) Acanthamoeba polyphaga is a possible host for Vibrio cholerae in aquatic environments. Exp Parasitol 126: 65-68

12. Abd H, Saeed A, Weintraub A, Nair GB, Sandström S (2007) Vibrio cholerae 01 strains are facultative intracellular bacteria, able to survive and multiply symbiotically inside the aquatic free-living amoeba Acanthamoeba castellanii. FEMS Microbiol Ecol 60: 33-39.

13. www.diss.kib.ki.se.

14. Abd H, Weintraub A, Sandström S (2005) Intracellular survival and replication of Vibrio cholerae $\mathrm{O} 139$ in aquatic free-living amoebae. Environ Microbiol 7 : 1003-1008.

15. Euzéby JP (1997) List of bacterial names with standing in nomenclature: a folder available on the internet. Int J Syst Bacteriol 47: 590-592.

16. http://emedicine.medscape.com/article/232038-overview

17. Popovic T, Olsvik $\varnothing$, Blake P A, Wachsmuth K (1993) Cholera in the Americas: Food borne aspects. J Food Protect 56: 811-821.

18. Kaper JB, Morris JG, Levine MM (1995) Cholera. Clin Microbiol Rev 8: 48-86.

19. Tauxe RV, Mintz ED, Quick RE (1995) Epidemic cholera in the new world: translating field epidemiology into new prevention strategies. Emerg Infect Dis 1: $141-146$.

20. Wachsmuth IK, Blake PA, Olsvik O (1994) Vibrio cholerae and cholera: Molecular to Global Perspectives. ASM Press, Washington, DC.
21. Sack DA, Sack RB, Nair, GB, Siddique AK (2004) Cholera. Lancet 363: 223 233.

22. Craig JP, Yamamoto K, Takeda Y, Miwatani T (1981) Production of a cholera-like enterotoxin by a Vibrio cholerae non-O1 strain isolated from the environment. Infect Immun 34: 90-97.

23. McLaughlin JC (1995) Vibrio. Manual of Clinical Microbiology. (6thedn), ASM Press, Washington.

24. Oates PJ, Touster O (1976) In vitro fusion of Acanthamoeba phagolysosomes. I. Demonstration and quantitation of vacuole fusion in Acanthamoeba homogenates. J cell Biol 68: 319-338.

25. Saeed A, Abd H, Edvinsson B, Sandstrom G (2009) Acanthamoeba castellani an environmental host for Shigella dysenteriae and Shigella sonnei. Arch Microbiol 191: 83-88.

26. Steinert M, Birkness K, White E, Fields B, Quinn F (1998) Mycobacterium avium bacilli grow saprozoically in coculture with Acanthamoeba polyphaga and survive within cyst walls. Appl Environ Microbiol 64: 2256-2261

27. Dalsgaard A, Serichantalergs O, Forslund A, Lin W, Mekalanos J, et al. (2001) Clinical and environmental isolates of Vibrio cholerae serogroup 0141 carry the CTX phage and the genes encoding the toxin-coregulatedpili. J Clin Microbio 39: 4086-4092.

28. Tobin-D'Angelo M, Smith AR, Bulens SN, Thomas S, Hodel M, et al. (2008) Severe diarrhea caused by cholera toxin-producing Vibrio cholerae serogroup O75 infections acquired in the southeastern United States. Clin Infect Dis 4 1035-1040.

29. Shinoda S, Nakagawa T, Shi L, Bi K, Kanoh Y, et al. (2004) Distribution of virulence-associated genes in Vibrio mimicus isolates from clinical and environmental origins. Microbiol Immunol 48: 547-551.

30. Sultan Z, Miyoshi S, Shinoda S (2006) Presence of LuxS/AI-2 based quorumsensing system in Vibrio mimicus: luxO controls protease activity. Microbio Immunol 50: 407-417.

31. Faruque SM, Albert MJ, Mekalanos JJ (1998) Epidemiology, genetics, and ecology of toxigenic Vibrio cholerae. Microbiol Mol Biol Rev 62: 1301-1314.

32. Blake PA, Allegra DT, Snyder JD, Barrett TJ, McFarland L, et al. (1980) Cholera -- a possible endemic focus in the United States. N Engl J Med 302: 305-309.

33. Weber JT, Mintz ED, Canizares R, Semiglia A, Gomez I, et al. (1994) Epidemic cholera in Ecuador. Multidrug-resistance and transmission by water and seafood. Epidemiol Infect 112: 1-11.

34. Colwell RR, Seidler RJ, Kaper J, Joseph SW, Garges S, et al. (1981) Occurrence of Vibrio cholerae serotype $\mathrm{O} 1$ in Maryland and Louisiana estuaries. App Environ Microbiol 41: 555-558.

35. Dobosh D, Gomez-Zavaglia A, Kuljich A (1995) The role of food in cholera transmission. Medicina 55: 28-32.

36. Mintz ED, Popovic T, Blake PA (1994) Transmission of Vibrio cholerae O1.Vibrio cholerae and cholera: Molecular to global perspectives. ASM press, Washington, DC.

37. Swerdlow DL, Greenea KD, Tauxe RV, Wellsa JG, Bean NH, et al. (1992) Waterborne transmission of epidemic cholera in Trujillo, Perú: lessons for a continent at risk. Lancet 340: 28-33.

38. Zychlinsky A, Sansonetti P (1997) Perspectives series: host/pathogen interactions. Apoptosis in bacterial pathogenesis. J Clin Invest 100: 493-495.

39. Kaysner CA (2000) The Microbiological Safety and Quality of Food. Aspen Publishers, Gaithersburg, MD.

40. Kaysner CA, Hill WE (1994) Toxigenic Vibrio cholerae O1 in food and water Vibrio cholerae and Cholera: New Perspectives on a Resurgent Disease. ASM Press, Washington, DC.

41. KirnTJ, Jude BA, Taylor RK (2005) A colonization factor links Vibrio cholerae environmental survival and human infection. Nature 438: 863-866.

42. PurdyA, Rohwer F, Edwards R, Azam F, Bartlett DH (2005) A glimpse into the expanded genome content of Vibrio cholerae through identification of genes present in environmental strains. J Bacteriol 187: 2992-3001.

43. Newsome AL, Scott TM, Benson RF, Fields BS (1998) Isolation of an Amoeba Naturally Harboring a Distinctive Legionella Species. Appl Environ Microbio 64: 1688-1693. 
Citation: Abd H, Shanan S, Saeed A, Sandström G (2011) Survival of Vibrio cholerae Inside Acanthamoeba and Detection of Both Microorganisms From Natural Water Samples May Point out the Amoeba as a Protozoal Host for V. cholerae. J Bacteriol Parasitol S1-003. doi:10.4172/21559597.S1-003

44. Cottingham KL, ChiavelliDA, Taylor RK (2003) Environmental microbe and human pathogen: the ecology and microbiology of Vibrio cholerae. Front Ecol Environ 1: 80-86.

45. Faruque SM., Naser IB, Islam MJ, Faruque AS, Ghosh AN, et al. (2005) Seasonal epidemics of cholera inversely correlate with the prevalence of environmental cholera phages. Proc Natl Acad Sci U S A 102: 1702-1707.

46. La Rocque RC, Krastins B, Harris JB, Lebrun LM, Parker KC, et al. (2008) Proteomic analysis of Vibrio cholerae in human stool. Infect Immun 76: 41454151.

47. Gelman BB, Rauf SJ, Nader R, Popov V, Borkowski J, et al. (2001) Amoebic encephalitis due to Sappinia diploidea. JAMA 285: 2450-2451.

48. Solomon JM, Rupper A, Cardelli JA, Isberg RR (2000) Intracellular growth of Legionella pneumophila in Dictyostelium discoideum, a system for genetic analysis of host-pathogen interactions. Infect Immun 68: 2939-2947.

49. Raper KB, Smith NR (1939) The Growth of Dictyostelium discoideum upon Pathogenic Bacteria. J Bacteriol 38: 431-445.

50. Thom S, Warhurst D, Drasar BS (1992) Association of Vibrio cholerae with fresh water amoebae. J Med Microbiol. 36: 303-306.

51. Weekers PH, Bodelier PL, Wijen JP, Vogels GD (1993) Effects of Grazing by the Free-Living Soil Amoebae Acanthamoeba castellanii, Acanthamoeba polyphaga, and Hartmannellavermi formison Various Bacteria. Appl Environ Microbiol 59: 2317-2319.

52. Axelsson-Olsson D, Waldenstrom J, Broman T, Olsen B, Holmberg M (2005) Protozoan Acanthamoeba polyphaga as a potential reservoir for Campylobacter jejuni. Appl Environ Microbiol 71: 987-992.

53. La Scola B, Raoult D (2001) Survival of Coxiella burnetii within free-living amoeba Acanthamoeba castellanii. ClinMicrobiol Infect 7: 75-79.

54. Barkers J, Humphrey TJ, Brown MW (1999) Survival of Escherichia coli 0157 in a soil protozoan: implications for disease. FEMS Microbiol Lett 173: 291-295.

55. Alsam S, Jeong SR, Sissons J, Dudley R, Kim KS, et al. (2006) Escherichia col interactions with Acanthamoeba: a symbiosis with environmental and clinical implications. J Med Microbiol 55: 689-94.

56. Abd H, Johansson T, Golovliov I, Sandström G, Forsman F (2003) Survival and growth of Francisella tularensis in Acanthamoeba castellanii. Appl Environ Microbiol 69: 600-606.

57. Winiecka-Krusnell J, Wreiber K, von Euler A, Engstrand L, Linder E (2002) Free-living amoebae promote growth and survival of Helicobacter pylori. Scand $\mathrm{J}$ Infect Dis 34: 253-256.

58. Cirillo JD, Falkow S, Tompkins LS (1994) Growth of Legionella pneumophila in Acanthamoeba castellanii enhances invasion. Infect Immune 62: 3254-3261.

59. Ly TM, Muller HE (1990) Ingested Listeria monocytogenes survive and multiply in protozoa. J Med Microbiol 33: 51-54.

60. King CH, Shotts EB, Wooley RE, Porter KG (1988) Survival of coliforms and bacterial pathogens within protozoa during chlorination. Appl Environ Microbiol 54: 3023-3033.

61. Jeong HJ, Jang ES, Han BI, Lee KH, Ock MS, et al. (2007) Acanthamoeba: Could it be an environmental host of Shigella? Exp Parasitol 115: 181-186.

62. Gaze WH, Burroughs N, Gallagher MP, Wellington EM (2003) Interactions between Salmonella typhimurium and Acanthamoeba polyphaga, and observation of a new mode of intracellular growth within contractile vacuoles. Microb Ecol 46: 358-369.

63. Knirel YA, Widmalm G, Senchenkova SN, Jansson PE, Weintraub A (1997) Structural studies on the short-chain lipopolysaccharide of Vibrio cholerae O139 Bengal. Eur J Biochem 247: 402-410.

64. Fritsche TR, Gautom RK, Seyedirashti S, Bergeron DL, Lindquist TD (1993) Occurrence of bacterial endosymbionts in Acanthamoeba spp. isolated from corneal and environmental specimens and contact lenses. J ClinMicrobiol 31 1122-1126.

65. Ismail N, Olano JP, Feng HM, Walker DH (2002) Current status of immune mechanisms of killing of intracellular microorganisms. FEMS MicrobiolLett 207: $111-120$.
66. Colwell RR, Kaper J, Joseph SW (1977) Vibrio cholerae, Vibrio parahaemolyticus, and other vibrios: occurrence and distribution in Chesapeake Bay. Science 198: 394-396

67. http://www.ncbi.nlm.nih.gov/books/NBK8526/

68. Alam M, Miyoshi S, Tomochika K, Shinoda S (1997) Hemagglutination is novel biological function of lipopolysaccharide (LPS), as seen with the Vibrio cholerae 0139 LPS. ClinDiagn Lab Immunol 4: 604-606.

69. Yamamoto T, Albert MJ, Sack RB (1994) Adherence to human small intestines of capsulated Vibrio cholerae O139. FEMS Microbiol Lett 119: 229-235.

70. Albert MJ, Qadri F, Bhuiyan NA, Ahmad SM, AnsaruzzamanM et al. (1999) Phagocytosis of Vibrio cholerae 0139 Bengal by human polymorphonuclear leukocytes. Clin Diagn Lab Immunol 6: 276-278.

71. Pettit DA, Williamson J, Cabral GA, Marciano-Cabral F (1996) In vitro destruction of nerve cell cultures by Acanthamoeba spp.: a transmission and scanning electron microscopy study. J Parasitol 82: 769-777.

72. Lu L, Walker WA (2001) Pathologic and physiologic interactions of bacteria with the gastrointestinal epithelium. Am J Clin Nutr 73: 1124-1130

73. Lock R, Ohman L, Dahlgren C (1987) Phagocytic recognition mechanisms in human granulocytes and Acanthamoeba castellanii using type 1 fimbriated Escherichia coli as phagocytic prey. FEMS Microbial Lett 44: 135 -140.

74. Tarsi R, Pruzzo C (1999) Role of surface proteins in Vibrio cholerae attachmen to chitin. Appl Environ Microbiol 65: 1348-1351.

75. Chiavelli DA, Marsh JW, Taylor RK (2001) The mannose-sensitive hemagglutinin of Vibrio cholerae promotes adherence to zooplankton. App Environ Microbiol 67: 3220-3225.

76. Bowers B, Korn ED (1969) The fine structure of Acanthamoeba castellanii (Neff strain). II. Encystment. J Cell Bio. 41: 786-805.

77. Hanna PC, Acosta D, Collier RJ (1993) On the role of macrophages in anthrax Proc Natl Acad Sci USA 90: 10198-10201.

78. Gao LY, Abu Kwaik Y (2000) The mechanism of killing and exiting of protozoan host Acanthamoeba polyphaga by Legionella pneumophila. Environ Microbiol 2: 79-90.

79. Johnson JA, Salles CA, Panigrahi P, Albert MJ, Wright AC, et al. (1994) Vibrio cholerae $\mathrm{O} 139$ synonym Bengal is closely related to Vibrio cholerae EI Tor but has important differences. Infect Immune 62: 2108-2110.

80. Kochi SK, Collier RJ (1993) DNA fragmentation and cytolysis in U937 cells treated with diphtheria toxin or other inhibitors of protein synthesis. Exp Cell Sci 208:296-302

81. Saeed A, Abd H, Edvinsson B, Sandström G (2007) Vibrio choleraeAcanthamoeba castellanii interaction showing Endosymbiont-Host relation. Symbiosis 44: 53-158.

82. Vezzulli L, Pruzzo C, Anwar Huq H, Colwell R (2010) Environmental reservoirs of Vibrio cholerae and their role in cholera. Environ Microbiol Rep 2: 27-33.

83. Shanan S, Abd H, Hedenström I, Saeed A, Sandström S (2011) Detection of Vibrio cholerae and Acanthamoeba species from same natural water samples collected from different cholera endemic areas in Sudan. BMC Res Notes 4 109

84. Shin OS, Tam VC, Suzuki M, Ritchie JM, Bronson RT, et al. (2011) Type II secretion is essential for the rapidly fatal diarrheal disease caused by non-O1, non-0139 Vibrio cholerae mBio 2: e00106-e00111.

85. Siddiqui R, Malik H, Sagheer M, Jung SY, Khan NA (2011) The type III secretion system is involved in Escherichia coli $\mathrm{K} 1$ interactions with Acanthamoeba. Exp Parasitol 128: 409-413.

86. Platz GJ, Bublitz DC, Mena P, Benach JL, Furie MB, et al. (2010) A tolC mutan of Francisella tularensis is hypercytotoxic compared to the wild type and elicits increased proinflammatory responses from host cells. Infect Immun 78: 1022 1031.

This article was originally published in a special issue, Microbial Communitie and Interactions handled by Editor(s). Dr. Lesouhaitier Olivier, University of Rouen, France; Dr. Louis Lambrechts, Institut Pasteur, France; Dr. Saber Gholizadeh, Urmia University, Iran. 